\title{
Eficacia y seguridad del peginterferón lambda para la Covid-19, indicios de un nuevo antiviral
}

\section{Efficacy and safety of peginterferon lambda for Covid-19, hints of a new antiviral}

https://doi.org/10.52808/bmsa.7e5.612.005

\author{
Eduardo Tuta-Quintero ${ }^{1, *}$ \\ https://orcid.org/0000-0002-7243-2238 \\ Camila Martinez Ayala ${ }^{2}$ \\ https://orcid.org/0000-0001-8275-4399 \\ Ana María Santos ${ }^{3}$ \\ https://orcid.org/0000-0002-1973-8043
}

Recibido: 06/04/2021

Aceptado: 22/05/2021

\section{RESUMEN}

Introducción: Los interferones son una familia de citocinas que actúan sobre la respuesta inmune innata del epitelio respiratorio, lugar de amplia invasión viral como el SARS-CoV-2. Objetivo: analizar la evidencia médica publicada sobre la eficacia y seguridad del interferón tipo III en el tratamiento de pacientes con COVID-19. Material y métodos: Revisión exploratoria de la literatura que incluyó PubMed hasta el 10 de marzo del 2021. Se incluyeron registros de ensayos clínicos de la Red de Registros de la organización mundial de la salud y publicaciones teóricas y publicaciones con datos empíricos en idioma español e inglés. Resultados: Se incluyeron 16 publicaciones y 4 registros de ensayos clínicos. Entre los documentos incluidos se encuentran revisiones de la literatura $(n=7)$, estudios in vitro $(n=4)$, ensayos clínicos aleatorizados ( $\mathrm{n}=2$ ), estudios in vivo $(\mathrm{n}=1)$, cartas al editor $(\mathrm{n}=1)$ y artículo de posición $(\mathrm{n}=1)$. Los 4 ensayos clínicos registrados se encontraron en U.S. National Library of Medicine. Conclusión: Únicamente se reporta un ensayo clínico publicado que muestran beneficios del interferón tipo III en pacientes ambulatorios con COVID-19. Cuatro registros de ensayos clínicos en curso permitirán conocer más sobre la eficacia y seguridad del PGL-1.

Palabras clave: Peginterferón lambda, Interferón, Interferón tipo III, COVID-19, SARS-CoV-2.

\begin{abstract}
Background: Interferons are a family of cytokines that act on the innate immune response of the respiratory epithelium, a site of widespread viral invasion such as SARS CoV-2. Objective: analyze the evidence medical published on the efficacy and safety of type III interferon in the treatment of patients with COVID-19. Material and methods: Exploratory review of the literature that included PubMed up to March 10, 2021. Clinical trial records from the World Health Organization Network of Registries and theoretical publications and publications with empirical data in Spanish and English were included. Results: 16 publications and 4 clinical trial registries were included. Among the documents included are literature reviews ( $=7)$, in vitro studies $(n=4)$, randomized clinical trials $(n=2)$, in vivo studies $(n=1)$, letters to the editor $(n=1)$ and position article $(n=1)$. Four registered clinical trials were found in the U.S. National Library of Medicine. Conclusion: Only one published clinical trial showing benefits of type III interferon in outpatients with COVID-19 is reported. Four clinical trial registries in progress will provide insight into the efficacy and safety of $P G L-1$.
\end{abstract}

Keywords: Peginterferon lambda, Interferon, Type III interferon, COVID-19, SARS-CoV-2.

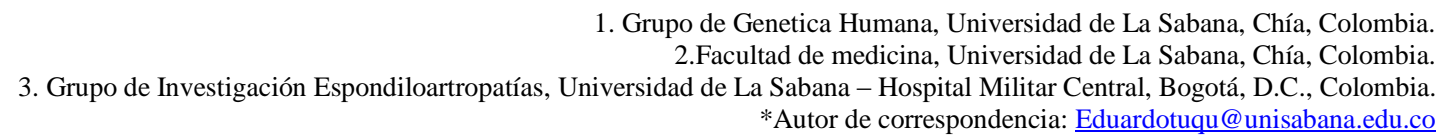

\section{Introducción}

En diciembre de 2019, surgió una extraña neumonía causada por un nuevo betacoronavirus conocido como síndrome respiratorio agudo severo coronavirus tipo 2 (SARS-CoV-2) en Wuhan, Hubei, China (Huang et al., 2020), tiempo después fue denominada 2019-nCoV (COVID-19), declarándose como una pandemia por la Organización Mundial de la Salud (Zhu et al., 2020). La presentación clínica de la COVID-19 varía desde una infección asintomática en el $80 \%$ de los casos, el $15 \%$ de los pacientes desarrollan neumonía grave que requiere hospitalización y soporte de oxígeno, y el $5 \%$ de ellos necesita ingreso en una unidad de cuidados intensivos (Guan et al., 2020). Los pacientes sintomáticos con enfermedad leve o moderada suelen presentar fiebre, tos, mialgia, cefalea, astenia/adinamia y anosmia (Huang et al., ob cit.; Guan et al., 2020). La respuesta inmune celular desencadenada por el reconocimiento de las células infectadas por el virus podría inducir una tormenta de citocinas y daño multiorgánico (Qin et al., 2020). La 
enfermedad severa se manifiesta con síndrome de dificultad respiratoria aguda (SDRA), shock séptico y/o falla multiorgánica, aumentando la mortalidad aproximadamente en el 7\%, pero si los individuos son inmunocomprometidos o adultos mayores de 65 años aumenta del 15 al 20\% (Zhu et al., 2020).

En la actualidad, el manejo médico antiviral utilizado en pacientes con Covid-19 como el lopinavir, un inhibidor de proteasas (Hurst \& Faulds, 2000), no ha demostrado resultados satisfactorios en términos de estancia hospitalaria, inicio de la ventilación mecánica y mortalidad (WHO et al., 2020). Únicamente, el remdesivir, análogo nucleósido de la adenosina (Eastman et al.,2020), y la dexametasona han demostrado en ensayos aleatorizados acelerar el tiempo de mejoría clínica en adultos hospitalizados con COVID-19 severo. Por otro lado, en la búsqueda de nuevas estrategias terapéutica que impactante favorablemente en los desenlaces clínicos, se ha estudiado la capacidad de los interferones (IFNs) para mantener una respuesta inmune antiviral adecuada en el epitelio respiratorio, se ha estudiado su rol en la replicación viral del SARS-CoV-2 en las células respiratorias, además, de regular la liberación de citocinas descontrolada "tormenta de citocinas" en las presentaciones clínicas más severas de la enfermedad (Hurst \& Faulds ob cit.; WHO et al., ob cit.).

Los IFNs son una familia de citocinas que se agrupan según su función y células que los producen: IFN tipo I (IFN $\alpha, \beta, \varepsilon, \kappa, \omega$, entre otros), tipo II (IFN $\gamma$ ), e INF tipo III (IFN $\lambda 1$, INF $\lambda 2$, IFN $\lambda 3$, IFN $\lambda 4$ ) (Schoggins, 2019). El sistema inmune innato del epitelio respiratorio es mediado por IFN tipo III y la expresión de genes que responden al IFN generando una respuesta antiviral, inmunomoduladora y antiproliferativas, resultando indispensables tanto las fisiopatologías y el control de las infecciones virales (Schoggins, 2019; Wells \& Coyne 2018). Omrani y colaboradores 11, compararon el tratamiento con INF-a-2a y ribavirina, un nucleótido mutagénico, para pacientes con infección grave por MERS-CoV, evidenciando una reducción de la carga viral y mejora en la supervivencia a los 14 días (Wells \& Coyne ob cit.). Debido a la similitud filogenética entre el SARS-CoV-2 y otros coronavirus, ha permitido que la comunidad científica busque identificar tratamientos basados en la respuesta inmune innata. Por lo cual, el peginterferón lambda 1 (PGL-1) en el manejo del SARS-CoV-2 podría ser un agente terapéutico eficaz en la infección de este patógeno; sin embargo, es escasa la información publicada. En esta revisión, se explora la evidencia médica disponible acerca de la eficacia y seguridad de la PGL-1 en pacientes con infección confirmada por SARS-CoV-2.

\section{Materiales y Métodos}

Se realizó una revisión sistemática exploratoria siguiendo los métodos propuestos por Arksey y O'Malley 12 y modificados por Levac 13: (i) definir la pregunta de investigación; (ii) buscar e identificar los estudios relevantes; (iii) graficar los datos; y (iv) resumir y reportar los resultados. Se respondió a la pregunta de investigación: ¿evidencia científica sobre la eficacia, efectividad y seguridad del PGL-1 en el tratamiento de pacientes con diagnóstico de COVID -19 ?

\section{Criterios de elegibilidad}

Se incluyeron i.) publicaciones teóricas (revisiones narrativas, comentarios, cartas al editor, artículos de posición) y publicaciones con datos empíricos (estudios experimentales, observacionales, in vivo e in vitro); ii). idioma inglés y español; iii). publicaciones que estudian o discuten la eficacia y perfil de seguridad del PGL-1 en la infección por SARS-CoV-2; iv). La revisión se limitó a documentos publicados entre los años 2019 a 2021. Se excluyeron guías de manejo clínico, protocolos de revisión y publicaciones sin acceso a resúmenes.

Selección de estudios y extracción de datos

Se realizaron estrategias de búsqueda para Pubmed y Scopus utilizando operadores booleanos idóneos para cada base de datos, los cuales fueron estructurados con la ayuda de un bibliotecólogo. La búsqueda inicial fue el 10 de marzo del año 2021 realizada por un autor (ET). Además, se generó una búsqueda de registros de ensayos clínicos en 17 bases de datos de la Red de Registros de la organización mundial de la salud OMS (WHO 2020), disponible en el archivo suplementario 1. Se realizaron reuniones periódicas con todos los autores generando un consenso sobre los documentos de texto completo y registro de ensayos clínicos que se incluyeron en la revisión. Para los documentos, se extrajeron los siguientes datos de los estudios elegibles: autores; tipo de estudio, objetivo, país de los autores y hallazgos principales; y en los registros de ensayos clínicos, sintetizamos en identificador del ensayo, el diseño del ensayo, el país, el tamaño de la muestra, grupo intervención y control, el resultado primario, la fecha de registro o fecha de inicio y la fecha de aproximada de finalización.

Síntesis y presentación de los resultados

La revisión se presenta en dos tablas para mostrar una descripción general de los estudios y registros de ensayos clínicos, seguido de una síntesis narrativa de los hallazgos más importantes, según lo establecido por Grudniewicz y colaboradores (2016). En este artículo, se empleó la extensión de la declaración PRISMA para reportar revisiones sistemáticas exploratorias (PRISMA-ScR) (Trico et al., 2018), archivo suplementario 2. 


\section{Resultados}

Se incluyeron 16 publicaciones y 4 registros de ensayos clínicos de la OMS (n=20) figura 1.

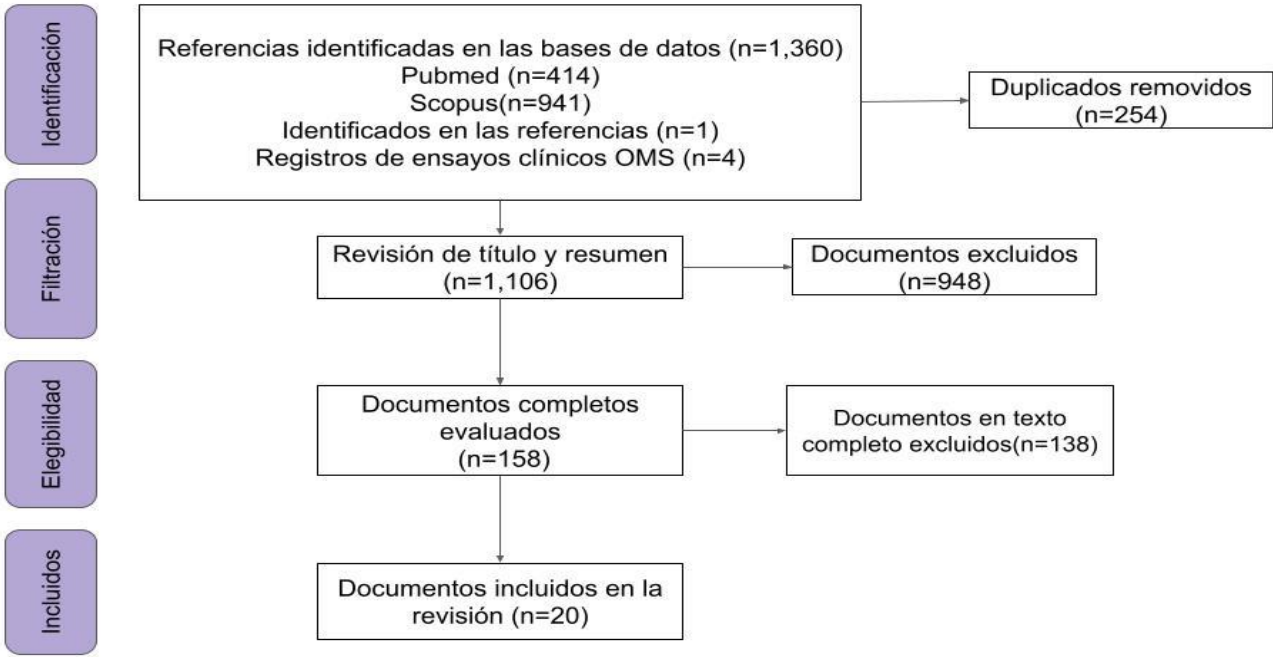

Figura 1. Flujograma PRISMA

Síntesis de los hallazgos de las publicaciones incluidas en la revisión

Entre los documentos incluidos se encuentran revisiones de la literatura $(n=7)$, estudios in vitro $(n=4)$, ensayos clínicos aleatorizados $(n=2)$, estudios in vivo $(n=1)$, cartas al editor $(n=1)$ y artículo de posición $(n=1)$. Todos los documentos fueron escritos en inglés. Los hallazgos principales de cada uno de estos documentos se describen en la tabla 1 .

\section{Ensayo clínico}

Feld y colaboradores (Feld et al., 2021), en un ensayo clínico aleatorizado controlado analizaron la seguridad y eficacia del PGL-1 en el tratamiento de pacientes ambulatorios con COVID-19 leve a moderado. Se administró PGL-1 de 180 microgramos $(\mathrm{mg})$ vía subcutánea a 30 pacientes en los primeros 7 días de aparición de los síntomas de covid o asintomáticos con hisopado positivo, se cuantificó carga viral siete días después de la intervención y se comparó con 30 pacientes a los cuales se les inyectó placebo. El estudio demostró una disminución de la carga viral en los pacientes del grupo intervención frente al placebo al día 7 (OR:4,12; IC del 95\% 1,15-16,73; p=0,029); se evidenció en ambos grupos una elevación de las aminotransferasa en forma leve y transitoria. El PGL-1 permite una rápida y marcada disminución en la carga viral en pacientes ambulatorios con infección por SARS-CoV-2, capaz de prevenir el deterioro clínico y acortar la duración de la diseminación viral con un alto perfil de seguridad.

Jagannathan et al. (2021), evaluaron la eficacia y seguridad de $180 \mathrm{mcg}$ de PGL-1 vía subcutánea a través de un ensayo clínico de fase 2, simple ciego, controlado y aleatorizado 1: 1 que estratificaba por edad ( $\geq 50$ y $<50$ años) y sexo con placebo en 120 pacientes con diagnóstico de COVID-19 ambulatorios. No se evidenciaron diferencias significativas en los niveles de la carga viral orofaríngea (HR: 0,51; IC del 95\% 0.26-1.04; $p=0,15$ ) y en el tiempo de duración de los síntomas (HR:0,94; IC 95\% 0,64-1,39; $\mathrm{p}=0,76$ ) en ambos grupos de estudio. Con respecto al perfil de seguridad, el $42 \%$ de los participantes del grupo intervención y 35\% del grupo placebo experimentaron eventos adversos, siendo la elevación de las enzimas hepáticas la manifestación más común reportada en el brazo de PGL-1 frente a placebo $(\mathrm{p}=0,027)$. Los autores mencionan que la administración de PGL-1 no disminuye el tiempo hasta de sintomatología clínica ni la carga viral en pacientes ambulatorios con COVID-19 no complicado.

\section{Estudios in vitro}

Los IFNs son producidos durante la infección y regulan genes estimulantes de IFNs que ayudan a limitar la replicación de los virus, sin embargo, en un estudio experimental de Busnadiego y colaboradores (2020), donde comparaban los INF I, II y III, se encontró que el IFN-III regulaba la inducción de los genes estimulados por IFN y actividad antiviral contra el SARS-CoV 2 en las células epiteliales bronquiales humanas primarias; adicionalmente, la 
capacidad del IFN tipo III de aumentar los niveles de la enzima convertidora de angiotensina 2 (ECA2) y expresión del receptor en la superficie celular es menor al compararse con los otros subgrupos. Los autores resaltan que la respuesta antiviral del IFN-III es más potente y se reduce los probables efectos generados por el aumento de la ECA2 y su receptor.

Tabla 1. Características de las publicaciones incluidas en nuestra revisión

\begin{tabular}{|c|c|c|c|c|c|}
\hline Autores & $\begin{array}{c}\text { Tipo de } \\
\text { documento }\end{array}$ & Objetivo & Revista & País & Hallazgo principal / contribución \\
\hline $\begin{array}{l}\text { Vanderheiden } \\
\quad \text { et al. }\end{array}$ & $\begin{array}{l}\text { Estudio in } \\
\text { vitro }\end{array}$ & $\begin{array}{l}\text { Determinar la respuesta inmune frente al } \\
\text { SARS-CoV-2 en cultivos de células del } \\
\quad \text { epitelio respiratorio humano }\end{array}$ & $\begin{array}{l}\text { Journal of } \\
\text { virology }\end{array}$ & $\begin{array}{l}\text { Estados } \\
\text { Unidos }\end{array}$ & $\begin{array}{l}\text { La respuesta inmune innata es mediada por IFN de tipo } \\
\text { I y III ante la infección por SARS-CoV-2 en células del } \\
\text { epitelio respiratorio humano }\end{array}$ \\
\hline Park et al. & $\begin{array}{l}\text { Revisión de } \\
\text { la literatura }\end{array}$ & $\begin{array}{l}\text { Capacidad antiviral mediadas por IFN tipo I } \\
\text { y III contra los coronavirus humanos y } \\
\text { potencial terapéutico }\end{array}$ & $\begin{array}{l}\text { Cell host and } \\
\text { microbe }\end{array}$ & $\begin{array}{l}\text { Estados } \\
\text { Unidos }\end{array}$ & $\begin{array}{c}\text { Respuesta inmune mediada por IFN tipo I y III es un } \\
\text { potencial objetivo terapéutico ante la infección por } \\
\text { SARS-COV-2 }\end{array}$ \\
\hline $\begin{array}{l}\text { Jagannathan } \\
\text { et al. }\end{array}$ & $\begin{array}{l}\text { Ensayo } \\
\text { clínico } \\
\text { aleatorizado }\end{array}$ & $\begin{array}{l}\text { Evaluar la seguridad y eficacia del PGL-1 en } \\
\text { el tratamiento de pacientes con COVID-19 } \\
\text { no complicado }\end{array}$ & Nat Commun & $\begin{array}{l}\text { Estados } \\
\text { Unidos }\end{array}$ & $\begin{array}{l}\text { El PGL-1 no produce un aclaramiento de la carga viral, } \\
\text { ni acorta la duración de la sintomatología clínica }\end{array}$ \\
\hline $\begin{array}{l}\text { Busnadiego } \\
\quad \text { et al. }\end{array}$ & $\begin{array}{l}\text { Estudio in } \\
\quad \text { vitro }\end{array}$ & $\begin{array}{l}\text { Investigar las propiedades antivirales de los } \\
\text { IFN tipo I, II y III asociado a la enzima } \\
\text { convertidora de angiotensina } 2\end{array}$ & mBio & Suiza & $\begin{array}{c}\text { Los IFN contra el SARS CoV } 2 \text { contrarrestan cualquier } \\
\text { efecto proviral de la inducción de la enzima } \\
\text { convertidora de angiotensina } 2\end{array}$ \\
\hline $\begin{array}{l}\text { Dinnon III y } \\
\text { cols }\end{array}$ & $\begin{array}{l}\text { Estudio in } \\
\text { vivo }\end{array}$ & $\begin{array}{l}\text { Evaluar las estrategias de tratamiento para la } \\
\text { COVID-19 en murinos }\end{array}$ & Nature & $\begin{array}{l}\text { Estados } \\
\text { Unidos }\end{array}$ & $\begin{array}{l}\text { El uso clínico de IFN tipo III como tratamiento para } \\
\text { COVID-19 podría ser benéficos en humanos }\end{array}$ \\
\hline $\begin{array}{l}\text { Felgenhauer } \\
\quad \text { et al. }\end{array}$ & $\begin{array}{l}\text { Estudio in } \\
\text { vitro }\end{array}$ & $\begin{array}{l}\text { Comparar las capacidades inhibitorias de los } \\
\text { IFN tipo I y III frente al SARS-CoV } 2\end{array}$ & J Biol Chem & Alemania & $\begin{array}{l}\text { SARS - CoV-2 es sensible a los IFN tipo I y III de } \\
\text { forma exógena, generando un posible candidato para el } \\
\text { manejo de la COVID-19 }\end{array}$ \\
\hline Feld et al. & $\begin{array}{l}\text { Ensayo } \\
\text { clínico } \\
\text { aleatorizado }\end{array}$ & $\begin{array}{l}\text { Evaluar la seguridad y eficacia del PGL-1 en } \\
\text { el tratamiento de pacientes con COVID-19 } \\
\text { leve a moderado }\end{array}$ & Lancet & Canadá & $\begin{array}{l}\text { El PGL-1 aumenta el aclaramiento viral, previene el } \\
\text { deterioro clínico y acorta la duración de la diseminación } \\
\text { viral }\end{array}$ \\
\hline $\begin{array}{l}\text { Jafarzadeh et } \\
\text { al. }\end{array}$ & $\begin{array}{l}\text { Revisión de } \\
\text { la literatura }\end{array}$ & $\begin{array}{c}\text { Describir la respuesta inmune mediada por el } \\
\text { INF del tipo I y de tipo III ante la infección } \\
\text { por SARS-COV-2 }\end{array}$ & $\begin{array}{c}\text { Viral } \\
\text { immunology }\end{array}$ & Irán & $\begin{array}{c}\text { IFN tipo III confieren inmunidad antiviral local a nivel } \\
\text { de las mucosas, sin inducir una respuesta pro } \\
\text { inflamatorias sistémicas }\end{array}$ \\
\hline Stanifer et al. & $\begin{array}{l}\text { Estudio in } \\
\text { vitro }\end{array}$ & $\begin{array}{c}\text { Describir el ciclo de vida del SARS-CoV } 2 \\
\text { en células epiteliales intestinales y describir } \\
\text { la respuesta inmune }\end{array}$ & Cell reports & Alemania & $\begin{array}{l}\text { Se evidenció una respuesta inmune mediada por IFN } \\
\text { tipo III capaz de controlar la replicación del SARS- } \\
\text { CoV-2 }\end{array}$ \\
\hline $\begin{array}{l}\text { Prokunina- } \\
\text { Olsson et al. }\end{array}$ & $\begin{array}{l}\text { Artículo de } \\
\text { posición }\end{array}$ & $\begin{array}{c}\text { Evaluar los beneficios del uso de IFN tipo III } \\
\text { para prevenir, limitar y tratar infecciones } \\
\text { virales }\end{array}$ & J Exp Med & $\begin{array}{l}\text { Reino } \\
\text { Unido }\end{array}$ & $\begin{array}{l}\text { IFN tipo III confieren inmunidad antiviral que podrían } \\
\text { ayudar en la actual pandemia }\end{array}$ \\
\hline Portela et al. & $\begin{array}{l}\text { Revisión de } \\
\text { la literatura }\end{array}$ & $\begin{array}{l}\text { Revisar la evidencia disponible sobre los IFN } \\
\text { tipo I y tipo III contra la infección por } \\
\text { SARS-CoV-2 }\end{array}$ & $\begin{array}{l}\text { Braz J Infect } \\
\text { Dis }\end{array}$ & Brazil & $\begin{array}{l}\text { IFN tipo III confieren inmunidad antiviral, considerado } \\
\text { para el tratamiento del COVID-19 }\end{array}$ \\
\hline Gaibani et al. & $\begin{array}{l}\text { Carta al } \\
\text { editor }\end{array}$ & $\begin{array}{l}\text { Evaluar el efecto de la terapia combinada } \\
\text { con interferón en pacientes críticos por } \\
\text { COVID-19 }\end{array}$ & $\begin{array}{l}\text { J Glob } \\
\text { Antimicrob } \\
\text { Resist }\end{array}$ & Italia & $\begin{array}{l}\text { Los pacientes críticamente enfermos que recibieron la } \\
\text { terapia, mostraron una ligera reducción en la carga viral } \\
\text { del SARS-CoV-2. }\end{array}$ \\
\hline Dowran et al. & $\begin{array}{l}\text { Revisión de } \\
\text { la literatura }\end{array}$ & $\begin{array}{c}\text { Exponer la capacidad antiviral relacionados } \\
\text { con la proteína transmembrana inducida por } \\
\text { IFN tipo III }\end{array}$ & Biochimie & Irán & $\begin{array}{l}\text { La proteína transmembrana inducida por IFN tipo III } \\
\text { desencadena propiedades antivirales frente a la } \\
\text { infección por SARS-CoV-2 }\end{array}$ \\
\hline Kow et al. & $\begin{array}{l}\text { Revisión de } \\
\text { la literatura }\end{array}$ & $\begin{array}{l}\text { Revisar las diferentes terapias basadas en } \\
\text { IFN para el tratamiento de pacientes con } \\
\text { COVID-19 }\end{array}$ & $\begin{array}{c}\text { Int } \\
\text { Immunopharma }\end{array}$ & Malaysia & $\begin{array}{c}\text { Pacientes con COVID-19 se podrían beneficiarse con } \\
\text { terapias intravenosa o inhalatoria de IFN en estadios } \\
\text { iniciales de la enfermedad }\end{array}$ \\
\hline Bagheri et al. & $\begin{array}{l}\text { Revisión de } \\
\text { la literatura }\end{array}$ & $\begin{array}{l}\text { Comparar terapia estándar para la COVID-19 } \\
\text { frente al manejo con IFN }\end{array}$ & $\begin{array}{c}\text { Int } \\
\text { Immunopharma }\end{array}$ & Irán & $\begin{array}{l}\text { IFN son candidatos en el tratamiento de COVID-19, sin } \\
\text { embargo, se debe evaluar su perfil de seguridad }\end{array}$ \\
\hline $\begin{array}{l}\text { Abdolvahab } \\
\quad \text { et al. }\end{array}$ & $\begin{array}{l}\text { Revisión de } \\
\text { la literatura }\end{array}$ & $\begin{array}{l}\text { Revisar la evidencia disponible sobre las } \\
\text { diferentes terapias con IFN para pacientes } \\
\text { con COVID-19 }\end{array}$ & $\begin{array}{c}\text { Int } \\
\text { Immunopharma }\end{array}$ & $\begin{array}{l}\text { Estados } \\
\text { Unidos }\end{array}$ & $\begin{array}{l}\text { La terapia con IFN apoyan la deficiencia inmunológica } \\
\text { en las fases avanzadas de la infección por SARS-CoV-2 }\end{array}$ \\
\hline
\end{tabular}

Notas: cols, colaboradores; IFN, interferón; SARS-CoV-2, síndrome respiratorio agudo severo coronavirus 2; PGL-1, peginterferon lambda 1; COVID-19, enfermedad por coronavirus 2019. 
Felgenhauer et al. (2020), evaluaron la capacidad inhibitoria del IFN tipo I y III durante 24 horas y potenciada por el ruxolitinib durante 16 horas en células Calu-3 de humano y Vero E6 de simio expuestas al SARS-CoV-2 y SARS-CoV-1. El PGL-1 a dosis de entre 10 a 100 ng/ml generó un efecto antiviral únicamente en las las células Vero E6 sobre el SARS-CoV-2, el ruxolitinib potenció la capacidad inhibitoria del PGL-1 debido probablemente a la vía de señalización antiviral janus quinasas (JAK), transductor de señal y activador de proteínas de transcripción (STAT). Por lo tanto, se concluyó una buena sensibilidad del SARS-CoV-2 a los IFN $\alpha$ y $\lambda$, este último asociado a una menor tasa de efectos adversos para el tratamiento de COVID-19.

Datos similares fueron reportados por Vanderheiden y colaboradores (2020), en donde compararon la respuesta entre el sistema inmunológico innato de barrera y citocinas pro-inflamatorias como la interleucina 6 (IL-6), el factor de necrosis tumoral alfa (TNF $\alpha$ ) e IFN tipo I y III; se utilizaron cultivos del epitelio de las vías respiratorias humanas en una interfaz aire-líquido. Encontrando que el SARS-CoV-2 infecta, se replica intracelular liberando citocinas proinflamatorias como IL-6 y TNF $\alpha$, que a su vez bloquean la producción de IFN de tipo I y III; sin embargo, al exponer el cultivo celular a los PGL-1 se logró una reducción en la replicación del virus. Los autores destacan su utilidad como opción terapéutica en pacientes con COVID-19.

\section{Estudios in vivo}

Dinnon III et al. (2020), alteraron genéticamente el dominio de unión al receptor del SARS-CoV-2 S para facilitar la unión eficiente al receptor ECA2 y replicarse en las células de las vías respiratorias superiores e inferiores de murinos con el fin de evaluar la eficacia y seguridad de terapia con PGL-1. Se administró por vía subcutánea 2 g de PGL-1 18 horas antes (profiláctico) y 12 horas después (terapéutico) de la infección con el virus. El manejo profiláctico y terapéutico protegieron a los murinos de la disfunción pulmonar y disminuyen la carga viral a nivel pulmonar en adultos menores de 1 año; sin embargo, a nivel del tracto respiratorio superior no se evidenció algún efecto sobre los títulos virales. El PGL-1 probablemente permite un mejor control y desenlaces clínicos dados por la infección por SARS-CoV-2 principalmente en murinos jóvenes.

Síntesis del registro de ensayos clínicos incluidas en la revisión

En el momento de la búsqueda, se encontraron cuatro ensayos clínicos registrados en U.S. National Library of Medicine, la suma de los participantes en los ensayos es de 490 individuos. Los hallazgos principales de cada uno de estos documentos se describen en la tabla 2.

Tabla 2. Características de los ensayos clínicos

\begin{tabular}{|c|c|c|c|c|c|c|c|c|}
\hline ID & Diseño del ensayo & País & $\begin{array}{c}\text { Tamaño } \\
\text { de } \\
\text { muestra }\end{array}$ & Intervención & $\begin{array}{c}\text { Control } \\
\dagger\end{array}$ & Ocurrencia primaria & $\begin{array}{l}\text { Fecha } \\
\text { inicio/ } \\
\text { registro }\end{array}$ & $\begin{array}{l}\text { Fecha } \\
\text { esperada } \\
\text { de fin }\end{array}$ \\
\hline NCT04331899 & $\begin{array}{c}\text { Ensayo clínico aleatorizado } \\
\text { controlado, paralelo, simple } \\
\text { ciego y abierto }{ }^{* *} \\
\text { Fase } 2\end{array}$ & EU & 120 & $\begin{array}{l}\text { Peginterferon } \\
\text { Lambda-1a }\end{array}$ & Placebo & $\begin{array}{l}\text { Conversión de la carga } \\
\text { viral del SARS-CoV-2 }\end{array}$ & $\begin{array}{l}\text { Abril de } \\
2020\end{array}$ & $\begin{array}{l}\text { Mayo de } \\
2021\end{array}$ \\
\hline NCT04344600 & $\begin{array}{c}\text { Ensayo clínico aleatorizado } \\
\text { controlado, paralelo y simple } \\
\text { ciego }{ }^{*} \\
\text { Fase } 2\end{array}$ & EU & 164 & $\begin{array}{l}\text { Peginterferon } \\
\text { Lambda-1a }\end{array}$ & Placebo & $\begin{array}{l}\text { Conversión de la carga } \\
\text { viral del SARS-CoV-2 }\end{array}$ & $\begin{array}{l}\text { Mayo de } \\
2020\end{array}$ & $\begin{array}{l}\text { Diciembre } \\
\text { de } 2021\end{array}$ \\
\hline NCT04354259 & $\begin{array}{c}\text { Ensayo clínico aleatorizado } \\
\text { controlado y paralelo } \\
\text { Fase } 2\end{array}$ & Canadá & 140 & $\begin{array}{c}\text { Brazo 1: } \\
\text { peginterferon } \\
\text { Lambda-1a } \\
\text { ambulatorio } \\
\text { Brazo 2: } \\
\text { peginterferon } \\
\text { Lambda-1a } \\
\text { hospitalizado }\end{array}$ & Placebo & $\begin{array}{l}\text { Conversión de la carga } \\
\text { viral del SARS-CoV-2, } \\
\text { reacción adversa } \\
\text { medicamentosa y tiempo } \\
\text { de resolución de síntomas }\end{array}$ & $\begin{array}{l}\text { Mayo de } \\
2020\end{array}$ & $\begin{array}{l}\text { Noviembre } \\
\text { de } 2021\end{array}$ \\
\hline NCT04388709 & $\begin{array}{c}\text { Ensayo clínico aleatorizado } \\
\text { controlado, paralelo y abierto } \\
\text { Fase } 2\end{array}$ & EU & 66 & $\begin{array}{l}\text { Peginterferon } \\
\text { Lambda-1a }\end{array}$ & Placebo & $\begin{array}{l}\text { Tiempo de resolución de } \\
\text { síntomas }\end{array}$ & $\begin{array}{l}\text { Septiembre } \\
\text { de } 2020\end{array}$ & $\begin{array}{l}\text { Mayo de } \\
2021\end{array}$ \\
\hline
\end{tabular}

Notas: *, reclutando; **, no reclutando; $\dagger$, Los detalles del manejo convencional no son descritos en los registros; SARS-CoV-2, síndrome respiratorio agudo severo coronavirus 2; COVID-19, enfermedad por coronavirus 2019.

El registro de ensayo clínico (NCT04344600) controlada fase II aleatorizado y ciego simple administrarán una única inyección subcutánea de PGL-1 de $180 \mathrm{mg}$ y comparándolos con pacientes no hospitalizados con alto riesgo de infección por SARS-CoV-2 en el hogar a quienes se les aplicó placebo; se hará un seguimiento durante un máximo de 
12 semanas posterior a la administración del PGL-1. El registro NCT04331899 es un ensayo abierto controlado aleatorio, y simple ciego, los pacientes serán asignados aleatoriamente 1: 1 a una dosis única de inyección subcutáneas de PGL-1 de 180 mg versus placebo en pacientes con enfermedad COVID-19 leve.

El registro NCT04354259 empleará dos brazos, una cohorte ambulatoria y otra hospitalizada. Los pacientes ambulatorios recibirán una única inyección subcutánea de PGL-1 de $180 \mathrm{mg}$ versus placebo, seguidos de forma remota con visitas para repetir el hisopado en los días 3 y 7. En la cohorte hospitalizada recibirán la misma dosis de PGL-1 a los días 0 y 7.

Los estudios NCT04344600 y NCT04354259 actualmente se encuentran reclutando los pacientes del estudio, a diferencia de NCT04388709 y NCT04331899 que aún no han comenzado con el reclutamiento. La totalidad de los registros evaluarán el tiempo en días de negativización de las cargas virales en dos muestras de las vías respiratorias superiores y resolución de los síntomas (fiebre, escalofríos, tos, congestión nasal, dolores musculares). El registro NCT04388709 evaluará adicionalmente la resolución de la hipoxia que requiere oxígeno suplementario para mantener la $\mathrm{SpO} 2>92 \%$ a los 7 días.

\section{Discusión}

En esta revisión sistemática exploratoria analizamos la actividad antiviral y seguridad del PGL-1 en pacientes con la infección por SARS-CoV-2. Hasta el momento, el PGL-1 cuenta con un ensayo clínico aleatorizado en 60 pacientes con la COVID-19 de leve a moderada que respalda la capacidad de disminuir la carga viral y prevenir el deterioro clínico con un alto perfil de seguridad (Feld et al., ob cit.); sin embargo, la evidencia es contradictoria al exponer un segundo ensayo clínico en 120 pacientes, en donde no se evidencia una reducción en la carga viral y disminución de los síntomas clínicos tras la administración del fármaco (Jagannathan et al., ob cit.). Estudios in vitro (Busnadiego et al., ob cit.; Felgenhauer et al., ob cit.: Vanderheiden et al., ob cit.: Dinnon et al., ob cit. y Sanford et al., ob cit.) e in vivo (Dinnon et al., 2020) reportan una sensibilidad del SARS-CoV-2 a los IFN tipo I y III y una respuesta inmunitaria mediada principalmente por el IFN $\lambda$ capaz de controlar la replicación viral. Por otro lado, se encontraron únicamente cuatro registros de ensayos clínicos investigando el rol del PGL-1 en la COVID-19, tanto en pacientes hospitalizados como ambulatorios. La totalidad de los registros son fase 2 y se encuentran registrados en U.S. National Library of Medicine, los estudios evaluarán la capacidad del fármaco para reducir la carga viral, resolución de síntomas clínicos y el perfil de seguridad del medicamento.

Si bien, los más recientes ensayos clínicos reportan resultados contrarios, con respecto a la disminución de la carga viral y duración de la sintomatología clínica en pacientes con COVID-19 no complicada (Feld et al., 2021; Jagannathan et al., ob cit.), se debe destacar la diferencia en el número de pacientes, la gravedad de la enfermedad y la terapia médica estándar entre ambos diseños de los estudios, lo cual pudo haber impacto en los diferentes resultados encontrados. Además, queda la duda si la capacidad antiviral del PGL-1 es más eficaz en las primeras etapas de la infección y en poblaciones cargas virales más altas, en donde la capacidad de respuesta del IFNs tipo III se ve desbordada por el SARS-CoV-2, limitando el control de la enfermedad por medio del sistema inmune innato (Portela Sousa \& Brites 2020; Andreakos et al.,2019; Kumagai et al.,2007).

La administración de formas recombinantes del IFN $\lambda$ o PGL-1 poseen una potente capacidad antiviral e inmunomodulación sobre la "tormenta de citocinas" que se puede presentar en la infección viral por SARS-CoV-2 24; además, se evidencia una estimulación de células $\mathrm{T}$ helper 1 , células $\mathrm{T}$ citotóxicas y anticuerpos, lo cuales son mediadores inmunes esenciales para desarrollar inmunidad a largo plazo (Prokunina-Olsson et al., 2020; Lazear et al., 2019; Jafarzadeh et al., 2020). Sin embargo, el inicio del manejo médico con PGL-1 debe interferir con el proceso inicial de la enfermedad antes de establecer una respuesta inmune descontrolada y lesión tisular multiorgánica, generando síntomas y el desarrollo de neumonía y / o SDRA. Esta premisa, partiendo desde la inmunidad natural de los IFNs tipo III que generan una protección óptima contra la infección y minimiza el daño tisular asociado a la infección, siendo el manejo médico temprano con PGL-1 una posible estrategia terapéutica que impacte favorablemente en la COVID-19 y sus desenlaces clínicos (Portela Sousa \& Brites ob cit.).

Un amplio espectro de virus desencadena una respuesta inmune desproporcionada en humanos, variando la severidad de los síntomas clínicos presentados en la enfermedad. La tormenta de citocinas o producción descontrolada de citocinas pro-inflamatorias como IL-6 y TNF- $\alpha$ y quimiocinas CXCL8 / IL-8 entre otras, contribuye al desequilibrio de la respuesta inmune innata y desenlaces clínicos desfavorable de la enfermedad, aumentando la morbimortalidad Portela Sousa \& Brites ob cit.; Lazear et al., ob cit.). Los IFNs tipo III son citocinas secretadas por células dendríticas, neutrófilos y células epiteliales de barrera (vías respiratorias y tracto gastrointestinal), que actúan sobre la respuesta inmune innata de las barreras de las superficies epiteliales, donde hay una amplia exposición a virus (Jafarzadeh et al., 2020). Por el contrario, a los IFNs tipo I que se expresan ampliamente y desencadenan una respuesta inflamatoria 
sistémica, los IFNs tipo III tiene la capacidad del control viral sin generar una activación sistémica del sistema inmune aguda exacerbada y daño tisular inflamatorio (Andreakos et al., ob cit.). Hasta el momento no es del todo claro si la respuesta mediada por IFNs están implicados en la exacerbación de la respuesta inflamatoria aguda en la infección por SARS-CoV-2, debido a que las vías de señalización se superponen y varias actividades antivirales funcionales se comparten entre sí (Andreakos et al., ob cit.; Kumagia et al., ob cit.).

Durante las infecciones virales, los IFNs son esenciales para combatir la replicación y diseminación viral por medio de la inducción en la cascada de señalización JAK/STAT y expresión de genes estimulados por IFNs (Portela Sousa \& Brites, ob cit.). En la actualidad, se lleva a cabo un ensayo clínico fase 2 evaluando el tratamiento del SDRA causado por COVID-19 con ruxolitinib, inhibidor de la transducción de señales de JAK-STAT, en 20 pacientes (NCT04334044); cuyo objetivo principal es la recuperación o cese de síntomas respiratorios durante 14 días. Si bien, el ruxolitinib en las células Calu-3 de humano potenció la capacidad antiviral del PGL-1, el cual, a su vez, desencadena una respuesta antiviral débil pero duradera por medio de la regulación positiva de los genes estimulados por IFNs en las células del epitelio respiratorio humano (Pervolaraki et al., 2018 y Ye et al., 2019), el perfil de seguridad del inhibidor de la vía JAK/STAT como fármaco inmunomodulador debe evaluarse minuciosamente.

Además, Gaspari y colaboradores (2020), evaluaron los efectos antivirales y desenlaces clínicos del ruxolitinib en dos pacientes con COVID leve a moderado. La utilización $5 \mathrm{mg}$ dos veces al día durante más de 2 días generó la aparición de lesiones purpúricas en la piel de los miembros superiores y dorsal, trombocitopenia (de 141.000 a 61.000) e infección de tejidos profundos del brazo izquierdo en un hombre de 74 años de edad. Se reportó una infección de herpes labial en una mujer de 63 años con dosis de $5 \mathrm{mg}$ dos veces al día durante 3 días. Ante el limitado tiempo disponible para el desarrollo de nuevos fármacos antivirales seguros y eficaces en el manejo de la COVID-19, se han reutilizado y diseñado estrategias terapéuticas basadas en los medicamentos previamente empleados en enfermedades infectocontagiosas y no infecciosas, planteando el uso del ruxolitinib en conjunto con el PGL-1 como un posible manejo médico eficaz y seguro en pacientes con COVID-19, al aumentar la eliminación del virus y la producción de anticuerpos contra el SARS-CoV-2 (Omrani et al., 2014 y Neubauer et al., 2020).

Los efectos adversos asociados al uso del PGL-1 pueden superponerse con la sintomatología y lesión tisular de la infección por SARS-CoV-2, dificultando su análisis a través de los estudios prospectivos. Uno de los principales efectos adversos descritos por Feld y colaboradores, fue un aumento leve y transitorio de las enzimas hepáticas; sin embargo, debido a la elevada expresión del receptor de la ECA2 a nivel el tracto gastrointestinal, principalmente en los hepatocitos, pueden generar una lesión tisular hepática y simular los efectos adversos a nivel hepáticas causados por el PGL-1 (Feld et al., 2021); además, una respuesta inmune descontrolada, fármacos utilizados en la hospitalización y un estado de sepsis y shock debido a la enfermedad por COVID-19, le confieren al hígado una tasa de lesión tisular aguda entre el 14 al 53\% de los de los pacientes y entre el 2 al el 11\% pueden desarrollar una enfermedad hepática crónica subyacente (Jothimani et al., 2020).

El alto perfil de seguridad y la ausencia de toxicidad hematológica mejoran considerablemente la tolerabilidad y el uso del PGL-1 en comparación con la de los interferones de tipo I o las manifestaciones extrapulmonares de la COVID-19 como linfopenia, trombocitopenia, trastorno trombóticos, anticuerpos antifosfolípidos entre otros (Lai et al.,2020). Previos estudios en otras infecciones virales habían determinado el perfil de seguridad del tratamiento con PGL-1; Chan y colaboradores (2016), evaluaron la actividad antiviral del PGL-1 frente al IFNs tipo I en pacientes con infección crónica por VHB. Se evidencio una disminución en el ADN-VHB y HBsAg hasta la semana 24 y menores tasas de efectos adversos (citopenias, astenia, adinamia y mialgias) en el grupo bajo tratamiento con PGL-1.

\section{Limitaciones y fortalezas}

Únicamente se incluyeron dos bases de datos para la búsqueda de información: PubMed y Scopus. Las estrategias de búsqueda de ambas bases fueron respaldadas y estructuradas con la ayuda de un bibliotecólogo para mejorar el mapeo del restringido número de evidencia disponible y a la ausencia de estudios clínicos que exploran las implicaciones clínicas del PGL-1 en la COVID-19. Nuestra revisión incluyó 17 bases de datos de registros clínicos. La guia PRISMA modificada para las revisiones sistemáticas exploratorias no incluyen una evaluación de la calidad de la evidencia, debido al amplio alcance temático de la pregunta de investigación (Arksey \& O’Malley, 2005; Levac et al., 2010; Tricco et al., ob cit). La pequeña población muestral incluida en los estudios limita los resultados clínicos esperados, siendo necesario un mayor número de ensayos clínicos aleatorizados y pacientes en los diferentes subgrupos etarios y comorbilidades.

\section{Conclusión}

Los IFNs de tipo III son potentes citoquinas antivirales, antiinflamatoria, inmunomoduladora y capaces de reducir la lesión tisular asociadas a las infecciones virales. Dada la falta de una vacuna y manejo médico eficaz, el PGL1 o IFN tipo III recombinante podría generar una respuesta antiviral, inmunomoduladora y antiproliferativas en las 
infecciones virales, y ser considerado como un tratamiento más para la COVID-19. Hasta el momento, únicamente un ensayo clínico reporta beneficios del PGL-1 en pacientes ambulatorios con COVID-19, reduciendo la carga viral del SARS-CoV-2, prevenir el deterioro clínico y acortar la duración de la diseminación viral con un alto perfil de seguridad. El compromiso hepático asociado a la elevación de las transaminasas fue transitorio y autorresolutivo; sin embargo, la lesión tisular asociadas a la infección puede sobreponerse o simular este efecto adverso. Cuatro registros de ensayos clínicos en curso permitirán conocer más sobre la eficacia y seguridad del PGL-1 en poblaciones con diferentes edades y comorbilidades infectadas por el SARS-CoV-2, además, de potenciales interacciones medicamentos. Consecuente al pequeño número de pacientes incluidos en los ensayos clínicos, son necesarios un mayor número de ensayos clínicos aleatorizados y pacientes en los diferentes subgrupos etarios y comorbilidades.

\section{Conflictos de intereses}

Ninguno para declarar.

\section{Agredecimento} logro.

Al Grupo de Genetica Humana, Universidad de La Sabana, y todas aquellas personas que colaboraron con este

\section{Referencias}

Andreakos E., Zanoni I. \& Galani I.E. (2019). Lambda interferons come to light: dual function cytokines mediating antiviral immunity and damage control. Curr Opin Immunol; 56:67-75. https://doi.org/10.1016/j.coi.2018.10.007.

Arksey H. \& O’Malley L. (2005). Scoping studies: towards a methodological framework. Int J Soc Res Methodol. 8:19-32. https://doi.org/10.1080/1364557032000119616.

Busnadiego I., Fernbach S., Pohl M.O., Karakus U., Huber M., Trkola A., Stertz S. \& Hale B.G. (2020). Antiviral Activity of Type I, II, and III Interferons Counterbalances ACE2 Inducibility and Restricts SARS-CoV-2. mBio;11(5): e01928-20. https://doi.org/10.1128/mBio.01928-20.

Chan H., Ahn S.H., Chang T.T., Peng C.Y., Wong D., Coffin C.S., Lim S.G., Chen P.J., Janssen H., Marcellin P., Serfaty L., Zeuzem S., Cohen D., Critelli L., Xu D., Wind-Rotolo M., Cooney E. \& LIRA-B Study Team (2016). Peginterferon lambda for the treatment of HBeAg-positive chronic hepatitis B: A randomized phase $2 b$ study (LIRA-B). Journal of hepatology. 64(5):1011-1019. https://doi.org/10.1016/j.jhep.2015.12.018.

Dinnon K.H. Leist S.R., Schäfer A., Edwards C.E., Martinez D.R., Montgomery S.A., West A., Yount B.L. Jr, Hou Y.J., Adams L.E., Gully K.L., Brown A.J., Huang E., Bryant M.D., Choong I.C., Glenn J.S., Gralinski L.E., Sheahan T.P. \& Baric R.S. (2020). A mouse-adapted model of SARS-CoV-2 to test COVID-19 countermeasures. Nature. 586(7830): 560-566. https://doi.org/10.1038/s41586-020-2708-8

Feld J.J., Kandel C., Biondi M.J., Kozak R.A., Zahoor M.A., Lemieux C., Borgia S.M., Boggild A.K., Powis J., McCready J., Tan D.H.S., Chan T., Coburn B., Kumar D., Humar A., Chan A., O'Neil B., Noureldin S., Booth J., Hong R., Smookler D., Aleyadeh W., Patel A., Barber B., Casey J., Hiebert R., Mistry H., Choong I., Hislop C., Santer D.M., Lorne Tyrrell D., Glenn J.S., Gehring A.J., Janssen H.L.A. \& Hansen B.E. (2021). Peginterferon lambda for the treatment of outpatients with COVID-19: a phase 2, placebo-controlled randomised trial. Lancet Respir Med. S2213-2600(20)30566-X. https://doi.org/10.1016/S2213-2600(20)30566$\underline{X}$.

Felgenhauer U., Schoen A., Gad H.H., Hartmann R., Schaubmar A.R., Failing K., Drosten C. \& Weber F. (2020). Inhibition of SARS-CoV-2 by type I and type III interferons. J Biol Chem. 295(41):13958-13964. https://doi.org/10.1074/jbc.AC120.013788.

Gaspari V., Zengarini C., Greco S., Vangeli V. \& Mastroianni A. (2020). Side effects of ruxolitinib in patients with SARS-CoV-2 infection: Two case reports. Int J Antimicrob Agents. 56(2):106023. https://doi.org/10.1016/j.ijantimicag.2020.106023.

Grudniewicz A., Nelson M., Kuluski K., Lui V., Cunningham H.V, X Nie J., Colquhoun H., Wodchis W.P., Taylor S., Loganathan M. \& Upshur, R.E. (2016). Establecimiento de objetivos de tratamiento para pacientes complejos: protocolo para una revisión del alcance. BMJ abierto. 6 (5), e011869. https://doi.org/10.1136/bmjopen-2016$\underline{011869}$.

Guan W.J, Ni Z.Y., Hu Y., Liang W.H., Ou C.Q., He J.X., W., Liu L., Shan H., Lei C., Hui D.S.C, Du B., Li L., Zeng G., Yuen K.Y., Chen R., Tang C., Wang T., Chen P., Xiang J., Li S., Wang J.L., Liang Z., Peng Y., Wei L., 
Liu Y., Hu Y-h., Peng P., Wang J.M., Liu J., Chen Z., Li G., Zheng Z., Qiu S., Luo J., Ye C., Zhu S. \& Zhong N. (2020). Clinical Characteristics of Coronavirus Disease 2019 in China. N Engl J Med.;382(18):1708-1720. https://doi.org/10.1056/NEJMoa2002032.

Huang C., Wang Y., Li X., Ren L., Zhao J., Hu Y., Zhang L., Fan G., Xu J., Gu X., Cheng Z., Yu T., Xia J., Wei Y., Wu W., Xie X., Yin W., Li H., Liu M., Xiao Y., Gao H., Guo L., Xie J., Wang G., Jiang R., Gao Z., Jin Q., Wang J. $\dagger \&$ Cao B. $\dagger$ (2020). Clinical features of patients infected with 2019 novel coronavirus in Wuhan, China. Lancet;395(10223):497-506. https://doi.org/10.1016/S0140-6736(20)30183-5.

Hurst M. \& Faulds D. (2020). Lopinavir. Drugs. 60(6):1371-9; discussion 1380-1. doi: 10.2165/00003495-20006006000009. PMID: 11152017.

Jafarzadeh A., Nemati M., Saha B., Bansode Y.D. \& Jafarzadeh S. (2020). Protective Potentials of Type III Interferons in COVID-19 Patients: Lessons from Differential Properties of Type I- and III Interferons. Viral Immunol. https://doi.org/10.1089/vim.2020.0076.

Jagannathan P., Andrews J.R., Bonilla H., Hedlin H., Jacobson K.B., Balasubramanian V., Purington N., Kamble S., de Vries C.R., Quintero O., Feng K., Ley C., Winslow D., Newberry J., Edwards K., Hislop C., Choong I., Maldonado Y., Glenn J., Bhatt A., Blish C., Wang T., Khosla C., Pinsky B.A., Desai M., Parsonnet J. \&, Singh U. (2021). Peginterferon Lambda-1a for treatment of outpatients with uncomplicated COVID-19: a randomized placebo-controlled trial. Nat Commun. 12(1):1967. https://doi.org/10.1038/s41467-021-22177-1.

Jothimani D., Venugopal R., Abedin M.F., Kaliamoorthy I. \& Rela M. (2020). COVID-19 and the liver. J Hepatol.73(5):1231-1240. https://doi.org/10.1016/j.jhep.2020.06.006.

Kumagai Y., Takeuchi O., Kato H., Kumar H., Matsui K., Morii E., Aozasa K., Kawai T., \& Akira S. (2007). Alveolar macrophages are the primary interferon-alpha producer in pulmonary infection with RNA viruses. Immunity. 27(2):240-252. https://doi.org/10.1016/j.immuni.2007.07.013.

Lai C.C., Ko W.C., Lee P.I., Jean S.S. \& Hsueh P.R. (2020). Extra-respiratory manifestations of COVID-19. Int J Antimicrob Agents;56(2):106024. https://doi.org/10.1016/j.ijantimicag.2020.106024.

Lazear H.M., Schoggins J.W. \& Diamond M.S. (2019). Shared and Distinct Functions of Type I and Type III Interferons. Immunity;50(4):907-923. https://doi.org/10.1016/i.immuni.2019.03.025.

Levac D., Colquhoun H. \& O’Brien K.K. (2010). Scoping studies: advancing the methodology. Implement Sci. 5:69. https://doi.org/10.1186/1748-5908-5-69.

Neubauer A., Wiesmann T., Vogelmeie, C.F., Mack E., Skevaki C., Gaik C., Keller C., Figiel J., Sohlbach K., Rolfes C., Renz H., Wulf H. \& Burchert A. (2020). Ruxolitinib for the treatment of SARS-CoV-2 induced acute respiratory distress syndrome (ARDS). Leukemia. 34(8): 2276-2278. https://doi.org/10.1038/s41375-020$\underline{0907-9}$.

Omrani A.S., Saad M.M., Baig K., Bahloul A., Abdul-Matin M., Alaidaroos A.Y., Almakhlafi G.A., Albarrak M.M., Memish Z.A. \& Albarrak A.M. (2014). Ribavirin and interferon alfa-2a for severe Middle East respiratory syndrome coronavirus infection: a retrospective cohort study. Lancet Infect Dis. 14(11):1090-1095. https://doi.org/10.1016/S1473-3099(14)70920-X.

Pervolaraki K., Rastgou Talemi S., Albrecht D., Bormann F., Bamford C., Mendoza J.L., Garcia K.C., McLauchlan J., Höfer T., Stanifer M.L., \& Boulant S. (2018). Differential induction of interferon stimulated genes between type I and type III interferons is independent of interferon receptor abundance. PLoS pathogens. 14(11):e1007420. https://doi.org/10.1371/journal.ppat.1007420.

Portela Sousa C. \& Brites C. (2020). Immune response in SARS-CoV-2 infection: the role of interferons types I and type III. Braz J Infect Dis. 24(5):428-433. https://doi.org/10.1016/j.bjid.2020.07.011.

Prokunina-Olsson L., Alphonse N., Dickenson R.E., Durbin J.E., Glenn J.S., Hartmann R., Kotenko S.V., Lazear H.M., O'Brien T.R., Odendall C., Onabajo, O.O., Piontkivska H., Santer D.M., Reich N.C., Wack A., \& Zanoni I. (2020). COVID-19 and emerging viral infections: The case for interferon lambda. The Journal of experimental medicine. 217(5):e20200653. https://doi.org/10.1084/jem.20200653.

Qin C, Zhou L, Hu Z, Zhang S, Yang S, Tao Y, Xie C., Ma K., Shang K., Wang W. \& Tian D.S. (2020). Dysregulation of Immune Response in Patients with Coronavirus 2019 (COVID-19) in Wuhan, China. Clin Infect Dis. 71(15):762-768. https://doi.org/10.1093/cid/ciaa248.

Sanford J. A., Nogiec C. D., Lindholm M. E., Adkins J. N., Amar D., Dasari S., Drugan J. K., Fernández F. M., RadomAizik S., Schenk S., Snyder M. P., Tracy R. P., Vanderboom P., Trappe S., Walsh M. J. \& Molecular 
Transducers of Physical Activity Consortium (2020). Molecular Transducers of Physical Activity Consortium (MoTrPAC): Mapping the Dynamic Responses to Exercise. Cell. 181(7):1464-1474. https://doi.org/10.1016/j.cell.2020.06.004.

Schoggins J.W. (2019). Interferon-Stimulated Genes: What Do They All Do? Annu Rev Viro. 6(1):567-584. https://doi.org/10.1146/annurev-virology-092818-015756.

Tricco A.C., Lillie E., Zarin W., O’Brien K.K., Colquhoun H. Levac D., Moher D., Peters M.D.J., Horsley T., Weeks L., Hempel S., Akl E.A., Chang C., McGowan J., Stewart L., Hartling L., Aldcroft A., Wilson M.G., Garritty C., Lewin S., Godfrey C.M., Macdonald M.T., Langlois E.V., Soares-Weiser K., Moriarty J., Clifford T., Tunçalp Ö. \& Straus S.E. (2018). PRISMA Extension for Scoping Reviews (PRISMA-ScR): Checklist and Explanation. Ann Intern Med. 169:467. https://doi.org/10.7326/M18-0850.

Tuta-Quintero E., Vega-Corredor M.C., Perdomo-Rodríguez L.S., \& Pimentel J. (2021). Colchicina, perspectivas de un viejo amigo para la reumatología en la COVID-19: una revisión exploratoria. Revista Colombiana de Reumatología, Advance online publication. https://doi.org/10.1016/j.rcreu.2021.02.0029.

Vanderheiden A., Ralfs P., Chirkova T., Upadhyay A.A., Zimmerman M.G., Bedoya S., Aoued H., Tharp G.M., Pellegrini K.L., Manfredi C., Sorscher E., Mainou B., Lobby J.L., Kohlmeier J.E., Lowen A.C., Shi P.Y., Menachery V.D., Anderson L.J., Grakoui A., Bosinger S.E. \& Suthar M.S. (2020). Type I and Type III Interferons Restrict SARS-CoV-2 Infection of Human Airway Epithelial Cultures. J Virol. 94(19): e00985-20. https://doi.org/10.1128/JVI.00985-20.

Wells A.I. \& Coyne C.B. (2018). Type III Interferons in Antiviral Defenses at Barrier Surfaces. Trends Immunol. 39(10):848-858. https://doi.org/10.1016/j.it.2018.08.008.

WHO Solidarity Trial Consortium, Pan H., Peto R., Henao-Restrepo A.M., Preziosi M.P., Sathiyamoorthy V., Abdool Karim Q., Alejandria M.M., Hernández García C., Kieny M.P., Malekzadeh R., Murthy S., Reddy,K.S., Roses Periago M., Abi Hanna P., Ader F., Al-Bader A.M., Alhasawi A., Allum E., Alotaibi A., Alvarez-Moreno C.A., Appadoo S., Asiri A., Aukrust P., Barratt-Due A., Bellani S., Branca M., Cappel-Porter H.B.C., Cerrato N., Chow T.S., Como N., Eustace J., García P.J., Godbole S., Gotuzzo E., Griskevicius L., Hamra R., Hassan M., Hassany M., Hutton D., Irmansyah I., Jancoriene L., Kirwan J., Kumar S., Lennon P., Lopardo G., Lydon P., Magrini N., Maguire T., Manevska S., Manuel O., McGinty S., Medina M.T., Mesa Rubio M.L., MirandaMontoya M.C., Nel J., Nunes E.P., Perola M., Portolés A., Rasmin M.R., Raza A., Rees H., Reges P.P.S., Rogers C.A., Salami K., Salvadori M.I., Sinani N., Sterne J.A.C., Stevanovikj M., Tacconelli E., Tikkinen K.A.O., Trelle S., Zaid H., Røttingen J.A. \& Swaminathan S. (2021). Repurposed Antiviral Drugs for Covid19 - Interim WHO Solidarity Trial Results. The New England journal of medicine, 384(6), 497-511. https://doi.org/10.1056/NEJMoa20231847. Eastman RT, Roth JS, Brimacombe KR, Simeonov A, Shen M, Patnaik S, et al. (2020). Remdesivir: A Review of Its Discovery and Development Leading to Emergency Use Authorization for Treatment of COVID-19. ACS Central Science; 6:672-83. https://doi.org/10.1021/acscentsci.0c00489.

World Health Organization (2020). WHO Registry Network. Disponible en: https://www.who.int/ictrp/network/primary/en/ (Acceso marzo 2021).

Ye L., Schnepf D. \& Staeheli P. (2019). Interferon- $\lambda$ orchestrates innate and adaptive mucosal immune responses. Nat. Rev. Immunol; 19, 614-625. https://doi.org/10.1038/s41577-019-0182-z.

Huang C., Wang Y., Li X., Ren L., Zhao J., Hu Y., Zhang L., Fan G., Xu J., Gu X., Cheng Z., Yu T., Xia J., Wei Y., Wu W., Xie X., Yin W., Li H., Liu M., Xiao Y., Gao H., Guo L., Xie J., Wang G., Jiang R., Gao Z., Jin Q., Wang J.† \& Cao B.† (2020). Clinical features of patients infected with 2019 novel coronavirus in Wuhan, China. Lancet;395(10223):497-506. https://doi.org/10.1016/S0140-6736(20)30183-5.

Zhu N., Zhang D., Wang W., Li X., Yang B., Song J., Zhao X., Huang B., Shi W., Lu R., Niu P., Zhan F., Ma X., Wang D., Xu W., Wu G., Gao G.F., Phil D. \& Tan W. (2020). A novel coronavirus from patients with pneumonia in China, 2019. N Engl J Med.; 24. https://doi.org/10.1056/NEJMoa2001017. 